\title{
ESTRUCTURA EN UNA TAXOCENOSIS DE LACERTIDAE (SAURIA, REPTILIA) DEL SISTEMA CENTRAL
}

\author{
Valentín Pérez Mellado'.
}

SUMMARY

A study on taxocenosis of Lacertidae with 8 species: Lacerta lepida, Lacerta schreiberi, Lacerta monticola, Acanthodactylus erythrurus, Psammodromus algirus, Psammodromus hispanicus, Podarcis hispanica and Podarcis bocagei in the western part of the Sistema Central (Iberian Peninsula) has been carried out.

Most of these behave as feeding generalists but $A$. erythrurus seems to specialize in mymecophagy whereas $L$. monticola turns out to be a «forced» sthenophage. The overlap values in each niche dimension suggest that the spatial dimension and habitat preference are the most important factors. However, segregation is produced jointly due to the interaction of the three dimensions considered which act interdependently.

\section{RESUMEN}

Se estudia una taxocenosis de lacertidae compuesta por 8 especies: Lacerta lepida, Lacerta schreiberi, Lacerta monticola, Acanthodactylus erythrurus, Psammodromus algirus, Psammodromus hispánicus, Podarcis hispánica y Podarcis bocagei.

La mayor parte de ellas se comportan como generalistas alimenticios, si bien $A$. erythrurus parece especializarse en la mirmecofagia mientras que $L$. monticola aparece como un estenófago «forzoso». Los va-

(1) Departamento de Zoología. Facultad de Biología. Universidad de Salamanca. 
lores de solapamiento en cada una de las dimensiones del nicho indican que la dimensión espacial y la preferencia de habitat actúan en primer término. Sin embargo la segregación se produce conjuntamente gracias a la interacción de las tres dimensiones consideradas que actúan independientemente.

\section{AGRADECIMIENTOS}

Quiero agradecer toda la ayuda prestada por mis compañeros de Departamento en la determinación de numerosas presas dudosas, especialmente a los Dres. V. Monserrat y E. Galante. Asimismo mi gratitud al Dr. A. Salvador por sus consejos y orientaciones y a F. Braña por las estimulantes discusiones. Por último, y muy especialmente, a mi mujer Pepa Carbonell por su constante apoyo.

\section{INTRODUCCIÓN}

Desde el estudio pionero de VALVERDE (1967) se ha prestado una creciente atención en la Península Ibérica a la estructura de las comunidades de Vertebrados. Si bien en el caso de los Reptiles, al margen de algunos estudios en curso de realización, sólo existe un trabajo publicado al respecto (MELLADO, et. al. 1975) sobre la comunidad de Saurios en Sierra Morena.

Es bien conocida la importancia herpetofaunística de las cadenas montañosas dentro de la región mediterránea por lo que hemos elegido un área del Sistema Central para nuestro estudio sobre la taxocenosis de la Familia Lacertidae.

Hemos preferido realizar un análisis por separado de dicha taxocenosis no incluyendo la totalidad de los Saurios presentes pues de este modo nos centramos sobre la porción de la comunidad en la cual se producen, presumiblemente, los fenómenos más notables de interacción y competencia potencial.

El estudio se ha llevado a cabo siguiendo el patrón ya clásico de división del nicho en sus tres dimensiones fundamentales: trófica, temporal y espacial (HUTCHINSON, 1957; PIANKA, 1978). 


\section{MATERIAL Y MÉTODOS}

Área estudiada.

Abarca las cadenas montañosas del área occidental del Sistema Central incluyendo las Sierras de Béjar, Francia, Gata y la Estrella en Portugal. La zona se incluye pues en el rectángulo con vértices en: $4010^{\prime} \mathrm{N}$, $4035^{\prime} \mathrm{N}$ y $0535^{\prime}, 0815^{\prime} \mathrm{W}$.

Se han llevado a cabo un total de 135 puntos de muestreo que abarcan toda la gama de habitats diferentes, con altitudes entre 450 y 2.440 metros. Las localidades fueron al menos visitadas en dos ocasiones en 1980 y 1981; los muestreos cubren todos los meses del año en cada uno de los diferentes medios (PÉREZ MELLADO, 1982).

Las características de altitud y humedad de la zona son propias para el desarrollo de distintos grados de vegetación (SCHMID, 1941; RIVAS GODAY, 1955) siendo dominante el grado Genista florida-Quercus pyrenaica RIVAS GODAY, si bien ligeras diferencias en la orientación o el nivel, provocan la aparición de condiciones mediterráneas más secas (PÉREZ MELLADO, 1982).

En cuanto a climatología, la zona se halla en un área de clima mediterráneo, si bien, en estas cadenas montañosas aparecen claras influencias continentales y atlánticas aumentando éstas últimas de Este a Oeste (OLIVER y LUIS CALABUIG, 1979). La zona ha sido denominada «área lluviosa del Sur» dentro del conjunto salmantino (LUIS CALABUIG, 1976) con dos máximos de precipitación, uno anterior y otro posterior a la estación estival. Las condiciones mediterráneas se acentúan de modo especial en ciertos valles como la cuenca del Alagón y Valle de Batuecas y en las laderas meridionales de las citadas Sierras.

Observaciones y Material estudiado.

Se han capturado un total de 1.125 ejemplares durante todos los meses del año y abarcando un período horario diario de las 07 a las 19 horas solares. La totalidad de ejemplares se conservó según los métodos usuales en Herpetología incluyendo la inyección del líquido fijador con objeto de paralizar rápidamente los procesos digestivos. 
La alimentación se estudió por análisis de contenidos gástricos incluyendo la totalidad de las presas presentes en toda la longitud del tubo digestivo. En algunas especies como L. monticola y L. lepida se han utilizado también algunos excrementos. En las dos especies del género Podarcis se analizaron además 25 contenidos gástricos conseguidos mediante regurgitación (LEGLER, 1977, 1979).

Las presas han sido determinadas a nivel Familia y en algunos casos hasta Género y Especie. La agrupación de los resultados en Órdenes obedece a un afán de simplificación con objeto de llevar a cabo con más facilidad las comparaciones interespecíficas. Ciertas Familias como Formicidae, dada su biología acusadamente diferente al resto de los Himenópteros, se han presentado aparte. La separación de Curculionidae se debe a su dominancia relativa dentro de la taxocenosis de Coleópteros.

\section{RESULTADOS}

Ocho especies de Lacertidae habitan el área de estudio:

Lacerta lepida DAUDIN, 1802; Lacerta schreiberi BEDRIAGA, 1878; Lacerta monticola BOULENGER, 1905; Acanthodactylus erythrurus SCHINZ, 1833; Psammodromus algirus LINNAEUS, 1758; Psammodrumus hispanicus FITZINGER, 1826; Podarcis hispánica STEINDACHNER, 1870; Podacis bocagei SEOANE, 1884.

Sus características biométricas, de folidosis y coloración han sido expuestas en otros lugares (PÉREZ MELLADO, 1981 y 1982).

Psammodromus algirus, Podarcis hispánica y Lacerta lepida se distribuyen en el área estudiada de modo general ocupando una amplia variedad de biotopos y sólo desaparecen al sobrepasar la cota de los 1.800 metros en P. hispánica y de 1.700 en PS. algirus y L. lepida.

$P$. bocagei ocupa fundamentalmente el piso del roble y sus etapas subseriales no exhibiendo el comportamiento saxícola característico en su congénere (PÉREZ MELLADO, 1981).

L. Monticola es una forma de montaña que, en el área estudiada, se distribuye desde los 1.700 metros hasta las más altas cotas (PÉREZ 


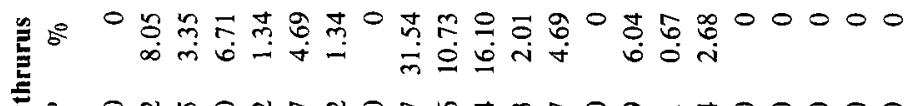

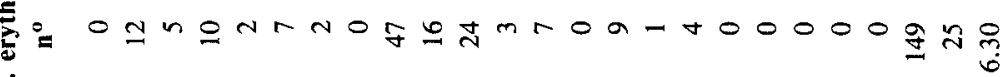

4

䒠

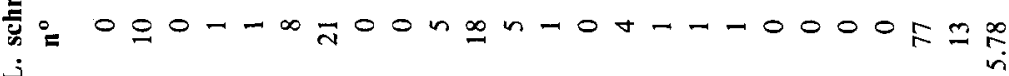

i

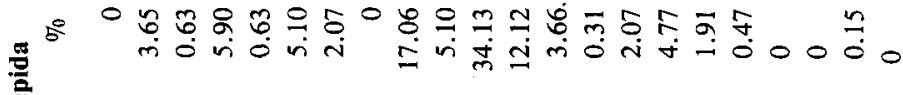

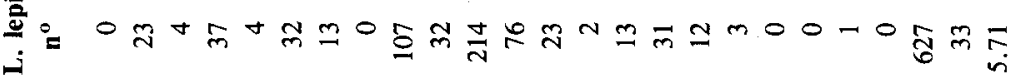

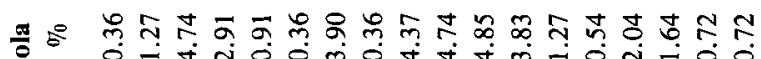

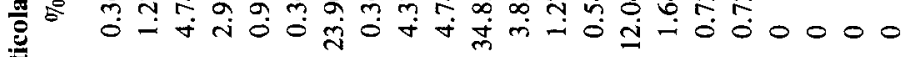

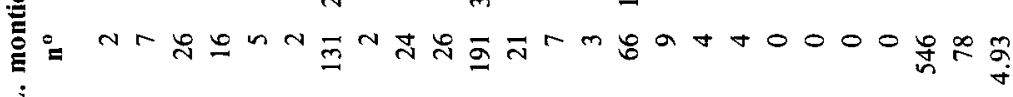

ذ

席

की

n $\stackrel{\infty}{\circ}$ 只

氙

灾:

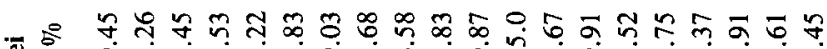

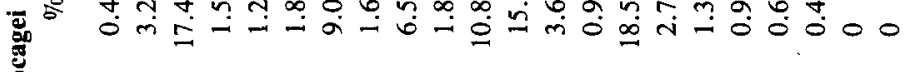

:

ะ

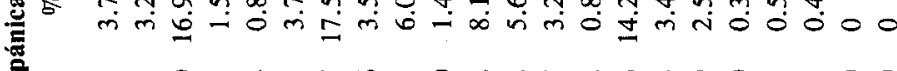

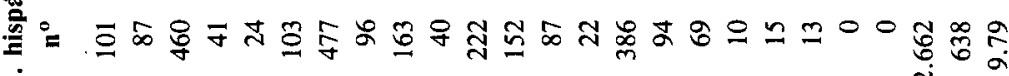

i

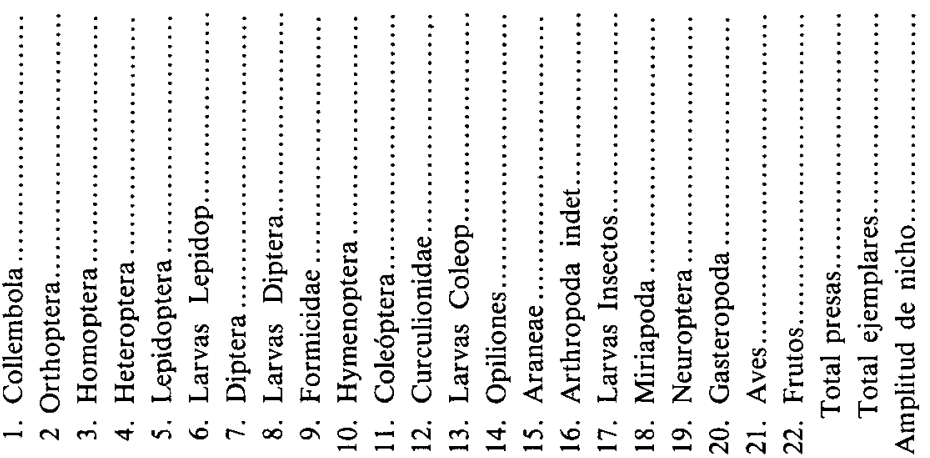


MELLADO, en prensa). L. schreiberi se acantona por su lado en zonas montañosas y valles con el único factor común de un grado relativamente elevado de humedad. Se presenta en el área en pequeñas poblaciones discontinuas y aisladas.

A. erythrurus y Ps. hispánicus son dos especies francamente escasas en la zona circunscribiéndose su presencia a los valles más acusadamente mediterráneos y a las vertientes meridionales de las Sierras donde ocupan enclaves bien definidos.

La gran heterogeneidad de las zonas montañosas hace difícil la cuantificación de una preferencia de biotopos (en el sentido de MELLADO, 1980) para cada una de las especies, sobre todo en el caso de formas sumamente eclécticas como $P$. hispánica y L. lepida. Hemos hallado, en efecto, todas las combinaciones de convivencia simpátrida entre las ocho especies salvo en el caso de las parejas $A$. erythrurus y L. monticola, así como L. monticola y PS. hispánicus.

\section{Dimensión trófica.}

Sobre un total de 1.125 tractos digestivos analizados se han determinado 5.469 presas de las cuales fueron medidas 2.610. En la Tabla 1 aparecen los resultados del análisis global para las ocho especies considerando 20 categorías taxonómicas de presas. En la mayoría de los casos existen notables diferencias en la dieta con relación al sexo y edad de los ejemplares así como la estación de que se trate (ver por ejemplo PÉREZ MELLADO, en prensa, para el caso de las dos Podarcis).

La amplitud de nicho trófico ha sido estimada, tanto a nivel de categorías taxonómicas, como de tamaño de presa, con la expresión de LEVINS (1968).

$$
A=\left(\sum P_{i}^{2}\right)^{-1}
$$

donde $\mathrm{p}_{\mathrm{i}}$ es la proporción de un determinado tipo de presa. A variará de la $n$ siendo $n$ el número de categorías consideradas. La utilización de A, en lugar de los índices derivados de la teoría de la Información (PIELOU, 1966; MARGALEF, 1958; SHANNON, 1948) tiene diver- 
sas ventajas (HESPENHEIDE, 1975) entre las cuales destacan su rapidez de cálculo y el hecho de que dicha expresión constituye el denominador de las fórmulas de solapamiento habitualmente utilizadas (ver también RUÍZ y JOVER, 1981).

Psammodromus algirus aparece, tanto en categorías taxonómicas como en clases de tamaño (ver Tabla 2) como la especie más eurífaga, seguida de ambas Podarcis. La mayor estenofagia de $P$. bocagei quizás esté relacionada con su también mayor especificidad de habitat (PÉREZ MELLADO, en prensa).

Las menores amplitudes de nicho corresponden a $L$. schreiberi y $L$. monticola, en el primer caso nuestros datos pueden tal vez desviarse de la realidad dado el exíguo tamaño de muestra. En cuanto a $L$. monticola la estenofagia parece una consecuencia obvia de habitar en zonas de alta montaña con una notable pobreza en la disponibilidad de recursos tróficos (PÉREZ MELLADO, en prensa).

La baja diversidad trófica de $L$. lepida, en cuanto a categorías taxonómicas se ve claramente compensada con la mayor amplitud de nicho a nivel de clases de tamaño depredadas, reflejo de una dieta diferencial en las distintas clases de edad del Lagarto ocelado (VALVERDE, 1967). Ps. hispánicus y A. erythrurus ocupan, desde el punto de vista trófico, situaciones intermedias en la taxocenosis, si bien la Lagartija colirroja posee una clara especialización en la captura de Himenópteros, fundamentalmente Formicidae.

\section{Dimensión temporal.}

Se han realizado un total de 1.466 observaciones horarias en las ocho especies. En la Tabla 3 aparecen los resultados de las mismas agrupados en 12 intervalos horarios que comienzan a las 07 horas y terminan a las 19, hora solar. Estos datos de actividad corresponden al total del período activo anual de cada una de las especies (ver Gráfica 1) en el área estudiada y deben, por tanto, tomarse como valores promedio pues es bien sabido que en los Saurios existen notables variaciones estacionales en los ritmos de actividad (ver por ejemplo SEVA y ESCARRÉ, 1980 y PÉREZ MELLADO, en prensa).

Los períodos de máxima actividad son aproximadamente coinci- 


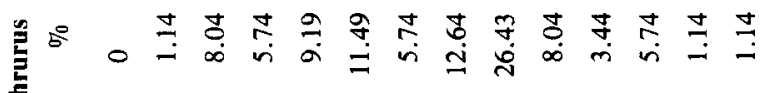

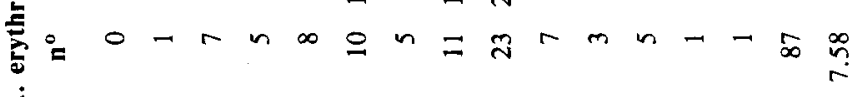

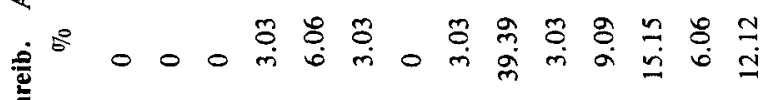

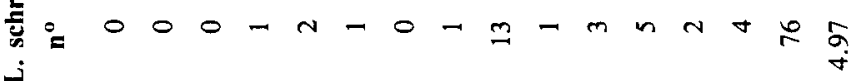

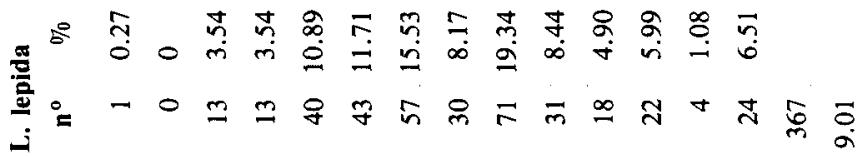

$$
\begin{aligned}
& \text { 章 }
\end{aligned}
$$

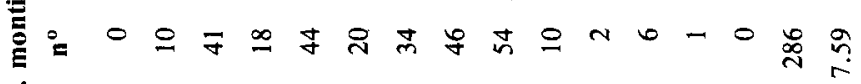

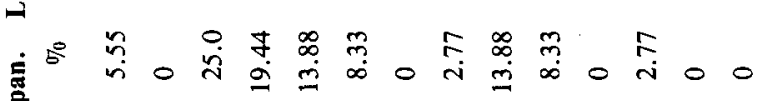

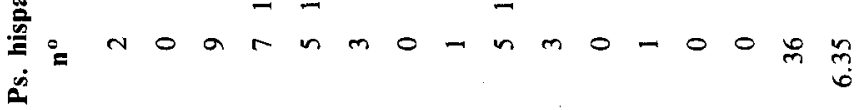

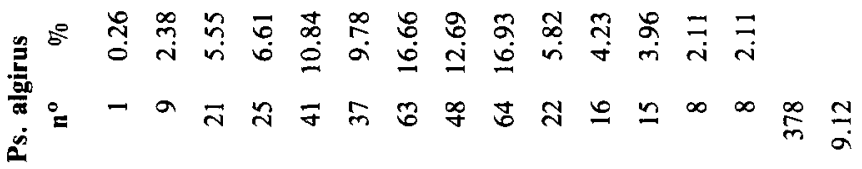

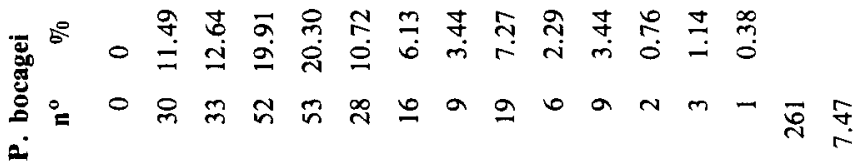

$$
\begin{aligned}
& \text { ๕ }
\end{aligned}
$$

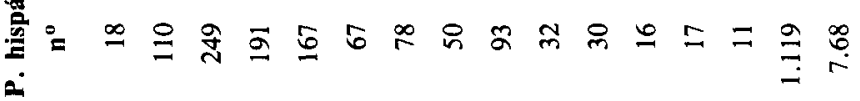




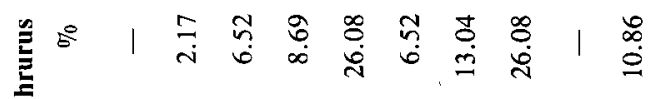

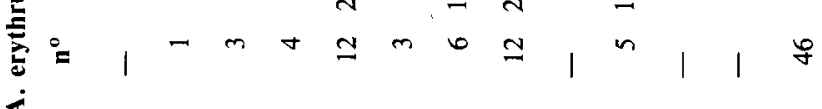

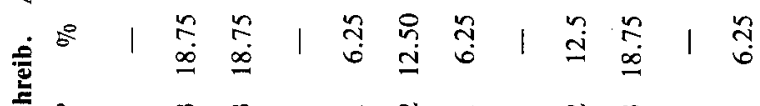

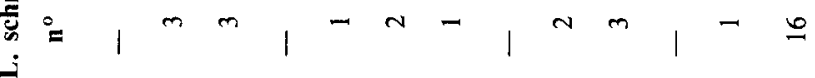

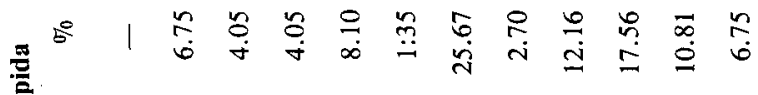

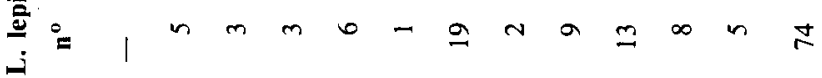

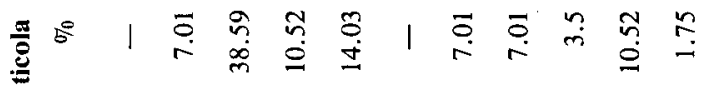

E

$\dot{-1}$

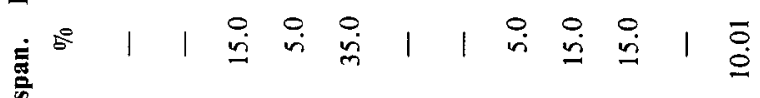

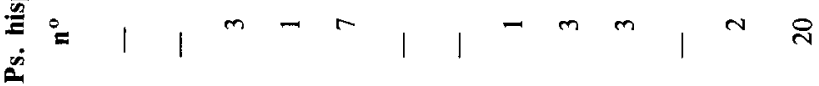

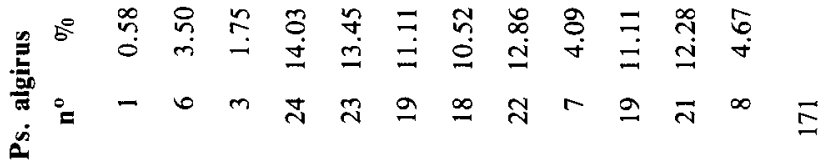

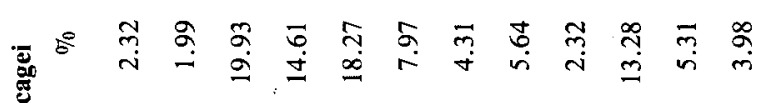

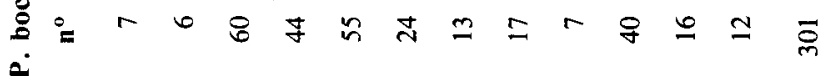

\#

:

a.

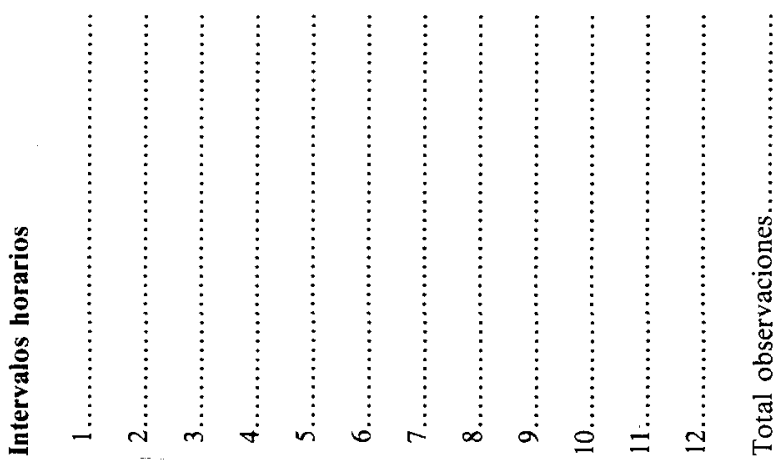




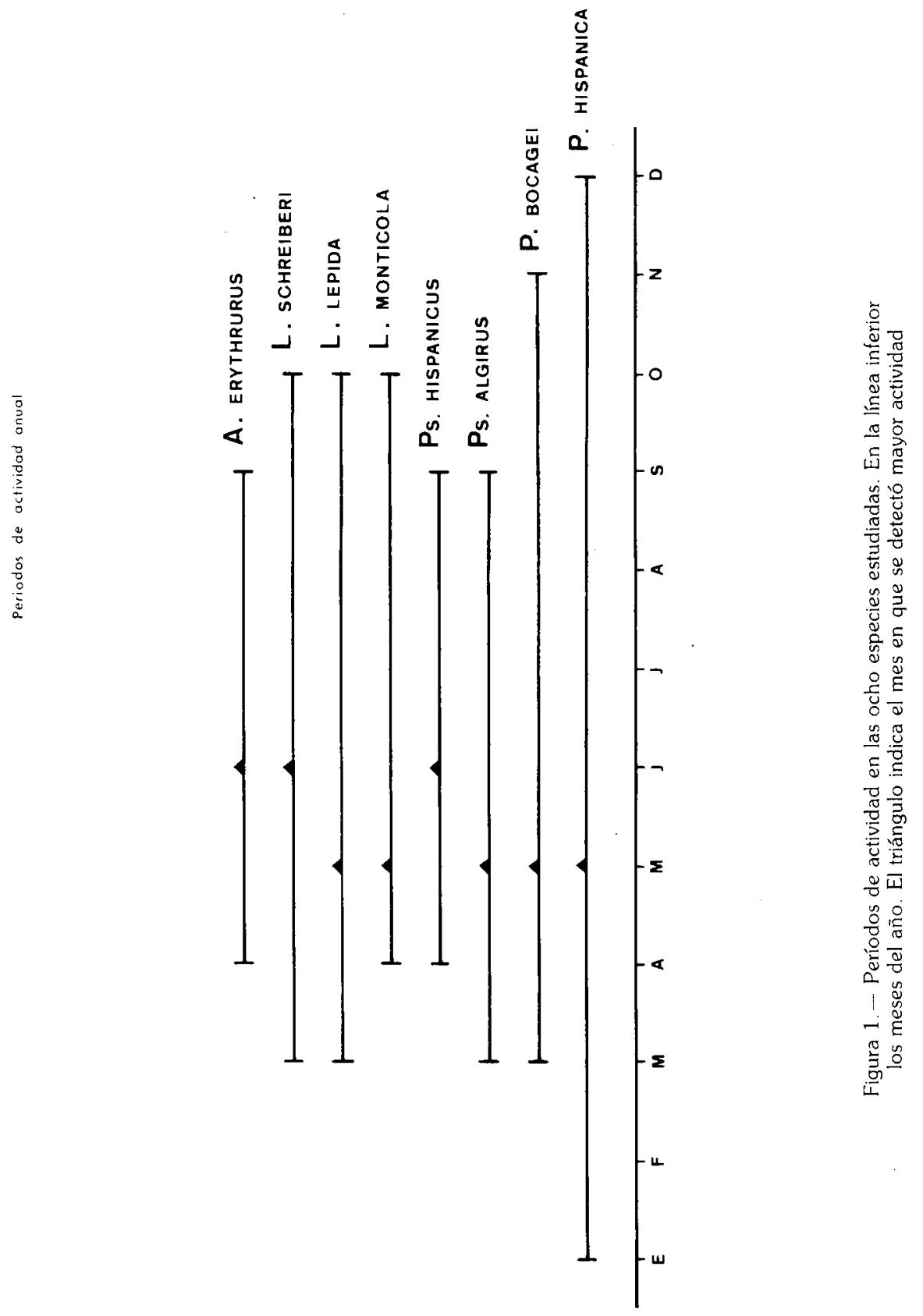


dentes en la mayoría de las especies ya que sus requerimientos térmicos son también similares (cf. BARBAULT, 1977).

\section{Dimensión espacial.}

Esta dimensión se ha estudiado en base a los datos proporcionados por un total de 684 observaciones. Se establecieron 6 categorías dentro de este capítulo:

1. Ejemplares observados sobre rocas o paredes rocosas a más de $20 \mathrm{~cm}$. del suelo.

2. Observados sobre suelo rocoso o rocas a menos de $20 \mathrm{~cm}$. del suelo.

3. Sobre suelo de tierra o con vegetación herbácea y cobertura vegetal arbustiva no superior al $50 \%$ de la superficie considerada.

4. Sobre plantas de menos de $20 \mathrm{~cm}$. de altura exhibiendo comportamiento trepador.

5. En el suelo de zonas arbustivas con más del $50 \%$ de cobertura vegetal.

6. Sobre troncos de árboles o sobre grandes matorrales, exhibiendo compartamiento trepador a más de $20 \mathrm{~cm}$. del suelo.

Esta clasificación tiene en cuenta dos aspectos, la dimensión vertical y la morfología del terreno. Las preferencias generales de habitat antes señaladas no entran habitualmente a formar parte de los cálculos de solapamiento.

HEATWOLE (1977) afirma que los Reptiles, en su selección de habitat, son más morfólogos que taxónomos o meteorólogos. Ello concuerda con nuestro enfoque de analizar la distribución espacial desde este punto de vista y no desde el de las comunidades vegetales presentes. El análisis fino de otros factores de la dimensión espacial del nicho corresponden más bien a los estudios autoecológicos (SEVA y ESCARRÉ, 1980). 


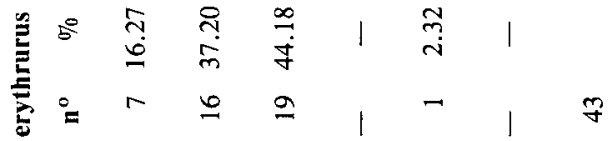

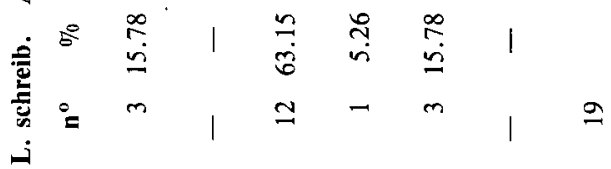

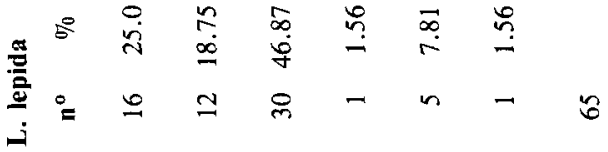

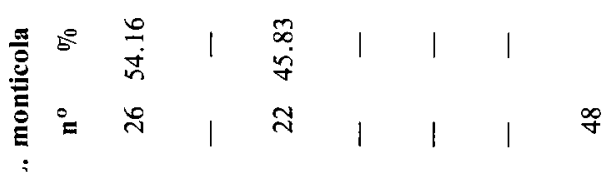

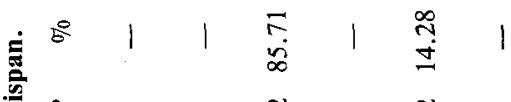

$$
\begin{aligned}
& \stackrel{\infty}{=}=1 \text { | }
\end{aligned}
$$

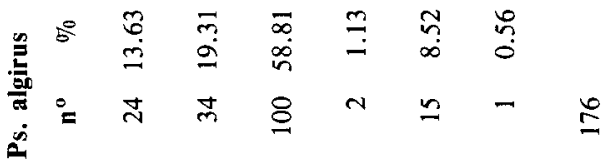

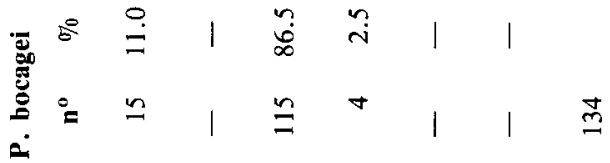

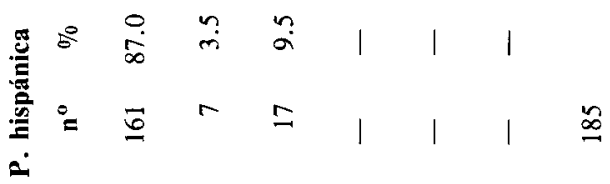

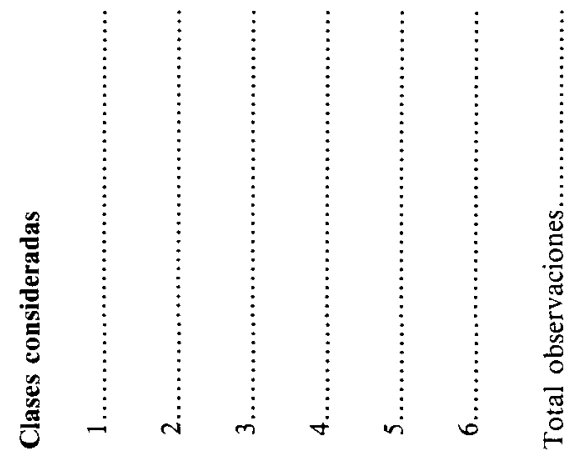


El mayor eclectismo en la distribución espacial corresponde a $L$. lepida y $P$ s. algirus confirmándose su gran adaptabilidad a una gran variedad de medios en la Península Ibérica (SALVADOR, 1974; ARNOLD y BURTON, 1978).

Solapamiento de nicho.

Para medir el grado de solapamiento entre cada una de las dimensiones del nicho así como los valores de solapamiento global hemos utilizado la fórmula de MACARTHUR y LEVINS (1967) y LEVINS (1968) simetrizada por MAY (1974) y ampliamente utilizada en estudios de comunidades reptilianas.

$$
S=\frac{\sum \mathrm{P}_{\mathrm{ij}} \cdot \mathrm{P}_{\mathrm{ik}}}{\sqrt{\sum \mathrm{P}_{\mathrm{ij}}{ }^{2} \cdot \mathrm{P}_{\mathrm{ik}}^{2}}}
$$

Donde $\mathrm{P}_{\mathrm{ij}}$ y $\mathrm{P}_{\mathrm{ik}}$ son las proporciones respectivas de las categorías $\mathrm{i}$ en las especies j y k. Se utiliza el solapamiento como tal y no en términos de «coeficiente de competencia» (ver diferencias conceptuales en PIANKA, 1974).

Las ocho especies solapan ampliamente en la dimensión trófica del nicho tanto en categorías taxonómicas como en clases de tamaño (ver Tablas 5 y 6). Existe de hecho una débil correlación positiva entre ambas subdimensiones $(r=0.20)$ sin significación estadística. Sin embargo se da una mayor segregación en la subdimensión del tamaño de presa ya que existe una correlación estadísticamente significativa entre ésta y los tamaños corporales medios de cada una de las especies ( $\mathrm{r}=$ $0.71 ; \mathrm{p}<0.05$, recta de regresión $\mathrm{y}=8.44 \mathrm{x}+11.44 ; \mathrm{r}^{2}=0.49 ;$ ver gráfica 2).

Parecen existir pues, diferencias entre los tamaños «preferidos» por unas y otras especies (la «diferencia de carácter» de SCHOENER, 1965, 1969; HESPENHEIDE, 1971, 1975; o «limitación de similitud» de MACARTHUR y LEVINS, 1967). Como era de esperar, también el número medio de presas por estómago está positivamente correlacionado con el tamaño corporal $(r=0.96 ; p<0.05$, Ver Tabla 7), si bien, como se observa en la Gráfica 3 en, al menos las tres especies con suficientes datos para este tipo de análisis, dicho número es bastante variable a lo 


\begin{tabular}{|c|c|c|c|c|c|c|c|c|}
\hline P. hispanica & P. hispanica & P. bocagei & Ps. algirus & Ps. hispanicus & A. erythrurus & L. lepida & L. schreiberi & L. monticola \\
\hline P. bocagei & 0.91 & & & & & & & \\
\hline Ps. algirus & 0.84 & 0.89 & & & & & & \\
\hline Ps. hispanicus & 0.70 & 0.89 & 0.80 & & & & & \\
\hline A. erythrurus & 0.46 & 0.50 & 0.63 & 0.32 & & & & \\
\hline L. lepida & 0.46 & 0.56 & 0.54 & 0.42 & 0.78 & & & \\
\hline L. schreiberi & 0.67 & 0.56 & 0.61 & 0.36 & 0.43 & 0.64 & & \\
\hline L. monticola & 0.72 & 0.66 & 0.53 & 0.44 & 0.51 & 0.77 & 0.87 & \\
\hline
\end{tabular}

Tabla 5. - Matriz de solapamiento trófico en la subdimensión de categorias taxonómicas depredadas

\begin{tabular}{|c|c|c|c|c|c|c|c|c|}
\hline & P. hispanica & P. bocagei & Ps. algirus & Ps. hispanicus & A. erythrurus & L. lepida & L. schreiberi & L. monticola \\
\hline P. bocagei & 0.94 & & & & & & & \\
\hline Ps. algirus & 0.67 & 0.71 & & & & & & \\
\hline Ps. hispanicus & 0.91 & 0.83 & 0.63 & & & & & \\
\hline A.erythrurus & 0.65 & 0.64 & 0.90 & 0.71 & & & & \\
\hline L. lepida & 0.60 & 0.63 & 0.97 & 0.59 & 0.92 & & & \\
\hline L. schreiberi & 0.35 & 0.35 & 0.64 & 0.44 & 0.82 & 0.73 & & \\
\hline L. monticola & 0.81 & 0.72 & 0.84 & 0.70 & 0.77 & 0.76 & 0.43 & \\
\hline
\end{tabular}

Tabla 6. - Matriz de solapamiento trófico en la subdimensión de clases de tamaño depredadas 


\section{Número medio de presas por estómago}

\begin{tabular}{|c|c|c|c|c|}
\hline & $\mathbf{x}$ & $\mathbf{s}$ & $\mathbf{n}$ & intervalo \\
\hline Ps. algirus.................... & 5.64 & 5.56 & 116 & $1-52$ \\
\hline Ps. hispánicus................ & 3.77 & 3.42 & 13 & $1-13$ \\
\hline L. monticola................ & 6.81 & 5.89 & 77 & $1-39$ \\
\hline L. lepida....................... & 21.10 & 27.05 & 29 & $3-100$ \\
\hline L. schreiberi................... & 8.44 & 7.63 & 9 & $1-26$ \\
\hline A. erythrurus................ & 9.26 & 7.92 & 19 & $2-36$ \\
\hline bocagei..................... & 3.96 & 3.63 & 153 & $1-13$ \\
\hline P. hispánica.................. & 4.85 & 5.71 & 570 & $1-59$ \\
\hline
\end{tabular}

Tabla 7. - Números medios de presa por estómago. Valores correspondientes a todo el período de estudio. ( $s=$ desviación típica)

\begin{tabular}{|c|c|c|c|c|c|c|c|c|}
\hline & P. hispanica & P. bosacer & Ps. alsirus & Ps hispanicus & A. erythrurus & 1. manticala & L. schreiber & L. lepids \\
\hline P. hispanica & & & & & & & & \\
\hline P. bocasel & 0.79 & & & & & & & \\
\hline Ps. alcirus & 0.86 & 0.79 & & & & & & \\
\hline Ps. hispanieus & 0.60 & 0.82 & 0.60 & & & & & \\
\hline A. erythrurus & 0.84 & 0.76 & 0.83 & 0.71 & & & & \\
\hline L. monticol:s & 0.70 & 0.89 & 0.50 & 0.65 & 0.59 & & & \\
\hline L. sehreiberi & 0.65 & 0.68 & 0.52 & 0.61 & 0.43 & 0.68 & & \\
\hline L.lepida & 0.77 & 0.56 & 0.71 & 0.52 & 0.76 & 0.47 & 0.64 & \\
\hline
\end{tabular}

Tabla 8. - Valores de solapamiento en la dimensión temporal del nicho 


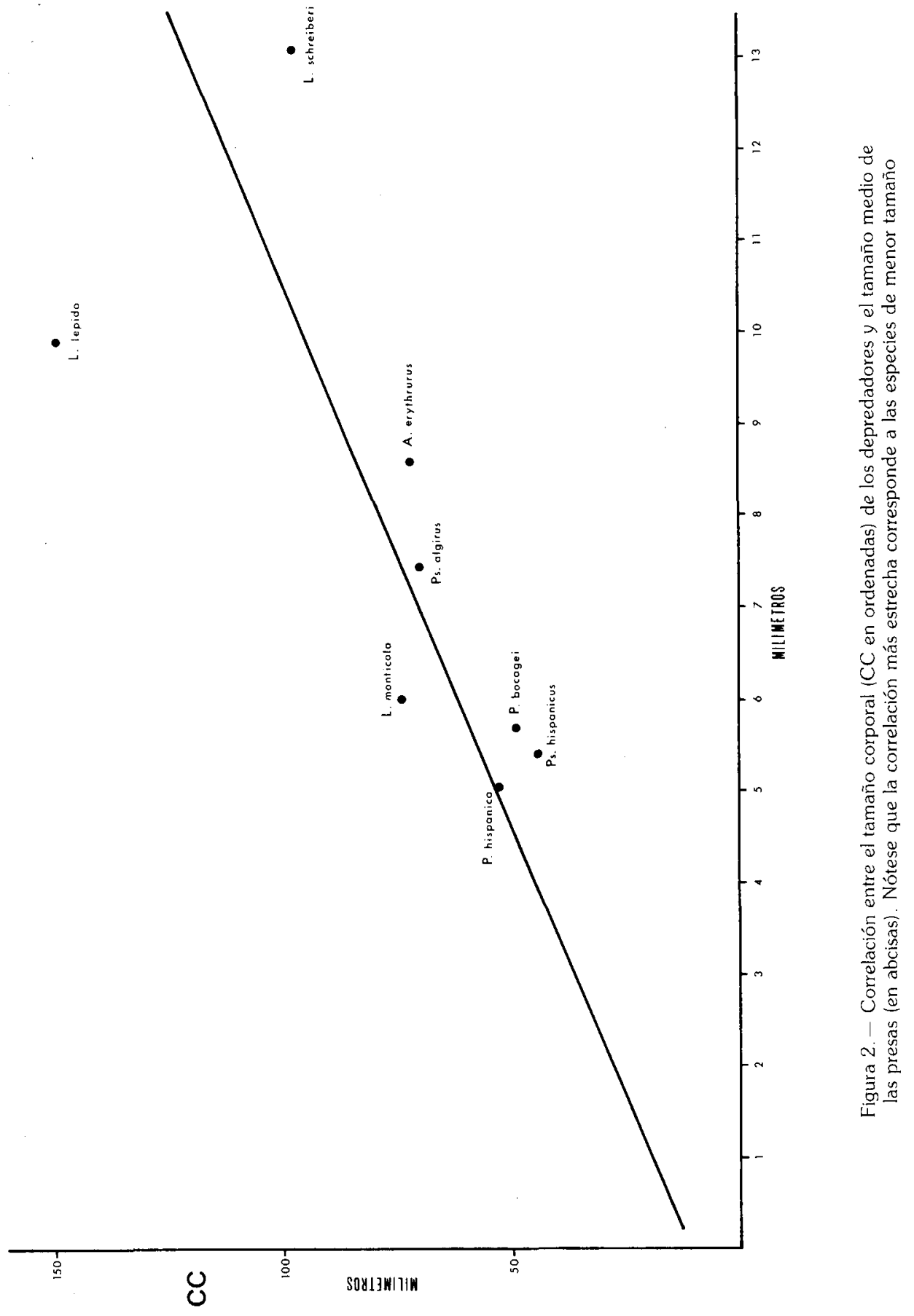



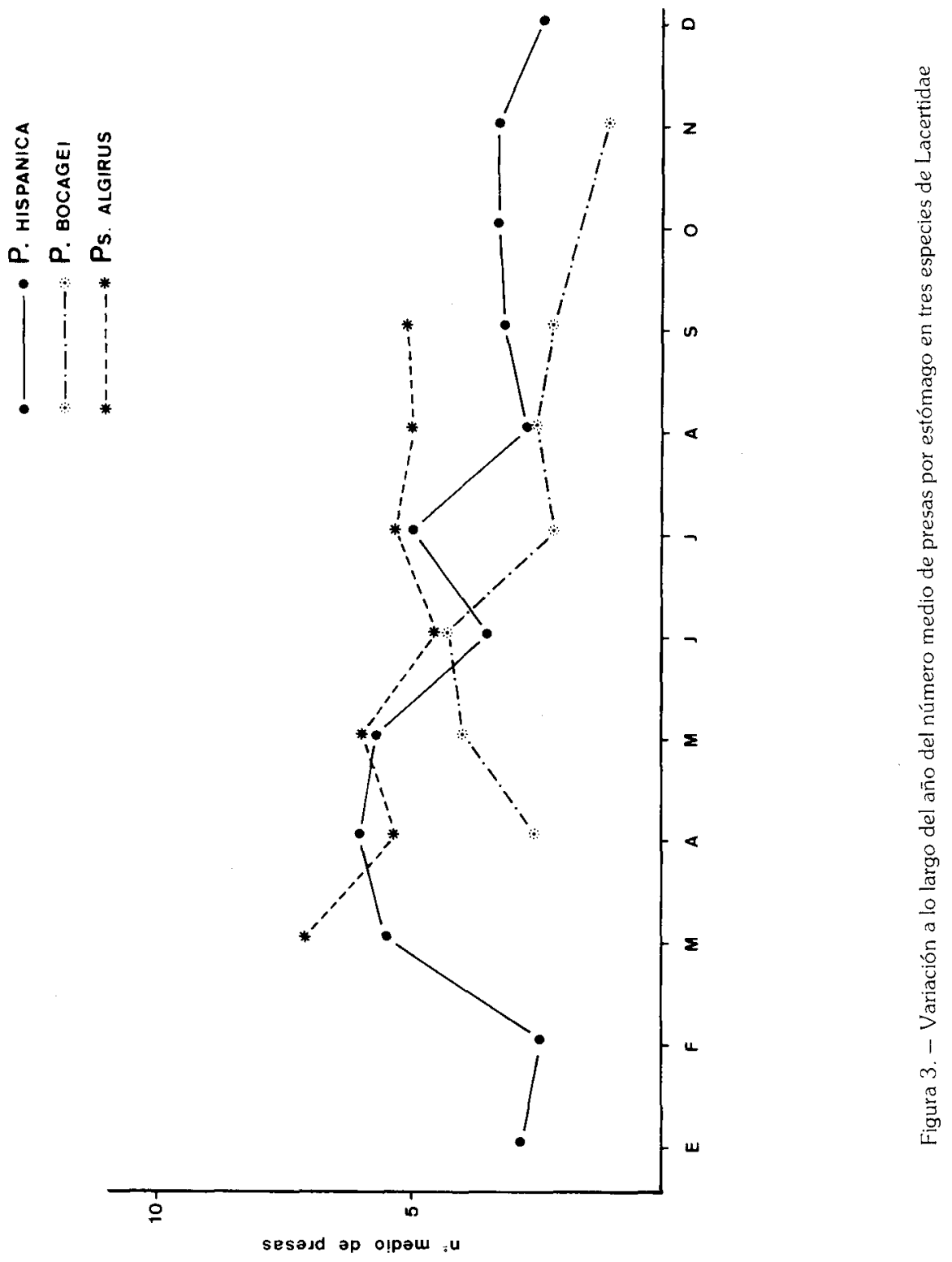
largo de las estaciones coincidiendo su máximo, aproximadamente, con los respectivos períodos reproductivos.

En la dimensión temporal del nicho las similares necesidades térmicas antes mencionadas dan como resultado un amplio solapamiento (ver Tabla 8) si bien L. monticola y L.schreiberi, en habitats con condiciones microclimáticas peculiares, se segregan ligeramente del resto.

La dimensión espacial, al igual que en otros estudios de Saurios (SCHOENER, 1974; MELLADO et. al., 1975) se revela como dimensión clave del nicho en la evitación de competencia interespecífica. Sobre todo en aquellas especies más emparentadas y por lo tanto con un más acusado parecido morfológico como $P$. hispánica y $P$. bocagei (ver Tabla 9 y PÉREZ MELLADO, 1981).

En nuestro caso no ha sido posible establecer, como hizo PIANKA (1969) para Ctenotus, correlaciones estadísticamente significativas entre la longitud relativa de los miembros posteriores y la utilización diferencial del nicho espacial debido, probablemente, a que trabajamos con una comunidad de Saurios en la que se incluyen varios Géneros diferentes en los cuales dicha longitud no sólo es una respuesta adaptativa a un nicho espacial determinado sino que además mantiene unas características inherentes al propio taxón considerado.

El sopamiento global puede finalmente medirse con dos métodos diferentes, cada uno de los cuales tiene sus propias limitaciones y ventajas (MAY, 1974; PIANKA, 1974). El método multiplicativo subestima la interacción real de los elementos que forman la comunidad (Tabla 10) ya que presupone la ortogonalidad de cada una de las dimensiones. El método de la media aritmética (Tabla 11) presupone una independencia total entre las tres dimensiones consideradas. Evidentemente, el caso de nuestra taxocenosis, y el de muchas otras comunidades, es intermedio entre ambas situaciones. En todo caso los valores encontrados indican solapamientos globales relativamente bajos y la existencia por tanto de mecanismos de segregación eficientes. Los mayores valores se verifican entre parejas de especies como $A$. erythrurus y $L$. lepida o bien Ps. hispánicus y $P$. hispánica, o bien Ps. algirus y $A$. erythrurus. En realidad y gracias a las preferencias de habitat características de Ps. hispánicus y $A$. erythrurus dicha interacción se ve considerablemente minimizada, máxime cuanto que estas dos especies aparecen de modo escaso en el área estudiada (ver más arriba). 


\begin{tabular}{|c|c|c|c|c|c|c|c|c|}
\hline & & & \\
\end{tabular}

Tabla 9. - Valores de solapamiento en la dimensión espacial del nicho

\begin{tabular}{|c|c|c|c|c|c|c|c|c|}
\hline & P. hispanica & P. bocagei & Ps. algirus & Ps. hispanicus & A. er ythrurus & L.monticola & L.schreiberi & L. lepida \\
\hline P. bocagei & 0.15 & & & & & & & \\
\hline$P_{S}$. algirus & 0.15 & 0.46 & & & & & & \\
\hline Ps. hispanicus & 0.04 & 0.58 & 0.16 & & & & & \\
\hline A. erythrurus & 0.09 & 0.18 & 0.43 & 0.11 & & & & \\
\hline L.monticola & 0.33 & 0.30 & 0.16 & 0.12 & 0.15 & & & \\
\hline L. schreiberi & 0.05 & 0.12 & 0.19 & 0.09 & 0.11 & 0.19 & & \\
\hline L. Iepida & 0.11 & 0.17 & 0.36 & 0.10 & 0.51 & 0.24 & & \\
\hline
\end{tabular}

Tabla 10. - Valores de solapamiento global con la multiplicación de los solapamientos parciales en cada dimensión 


\begin{tabular}{|c|c|c|c|c|c|c|c|c|}
\hline & $P$ hisparicar & P bocasos & $P_{s}$ alsirus & Ps hispanicus & A ervyliburus & 1 monticula & I schre ibern & 1 lepidata \\
\hline P hispanica & & & & & & & & \\
\hline P bocalaei & 0.71 & & & & & & & \\
\hline As altirus & 0.67 & 0.83 & & & & & & \\
\hline$P$ hissenicus & 0.58 & 0.87 & 0.64 & & & & & \\
\hline A erythrurus & 0.58 & 0.66 & 0.82 & 0.61 & & & & \\
\hline I monticola & 0.76 & 0.75 & 0.65 & 0.61 & 0.63 & & & \\
\hline L. schrabes, & 0.51 & 0.64 & 0.67 & 0.59 & 0.61 & 0.69 & & \\
\hline L Iepidz & 0.59 & 0.65 & 0.81 & 0.59 & 0.85 & 0.72 & 0.73 & \\
\hline & & & & & & & & \\
\hline
\end{tabular}

Tabla 11. - Valores de solapamiento global como medida aritmética de los solapamientos parciales en cada dimensión

\section{DISCUSIÓN Y CONCLUSIONES}

La taxocenosis estudiada está formada por especies, que salvo excepciones que ahora comentaremos, se comportan como generalistas alimenticios (EMLEN, 1973). Dentro de la comunidad parece existir un especialista, $A$. erythrurus que centra su depredación sobre los Himenópteros, especialmente Formicidae; su existencia sólo parece posible en el caso de que el recurso trófico explotado sea abundante (MACARTHUR y PIANKA, 1966) como ocurre en la Lagartija colirroja que habita en zonas con gran abundancia de Himenópteros. Según la teoría de las estrategias alimenticias el incremento de alimento induce la especialización trófica (SCHOENER, 1971; PIKE et. al., 1977). Además debe tenerse en cuenta que la captura de Himenópteros de buen tamaño supone un aporte energético considerable a la dieta de cualquier especie (SCHOENER, 1971). La existencia de un especialista mirmecófago en comunidades de Saurios ha sido puesta de manifiesto por otros autores (por ejemplo Phrynosoma modestum en el Desierto de Mapimi estudiado por BARBAULT, GRENOT y URIBE, 1978). 
Sin embargo existen otras causas para explicar la especialización trófica como es el caso de L. monticola, que habita un medio de escasos recursos, similares conclusiones extraen BALLINGER y BALLINGER (1979) al estudiar el comportamiento alimenticio de Sceloporus jarrovi (realizando además una aguda crítica de la teoría de SCHOENER, 1971).

El tamaño de presa aparece también como factor de segregación, si bien las discusiones en este aspecto han sido habitualmente enfocadas desde este único punto de vista, sin tener en cuenta el tipo de presa (HESPENHEIDE, 1971; SCHOENER, 1969) a pesar de que se reconoce la importancia empírica de este factor (ORIANS y HORN, 1969). HESPENHEIDE (1975) (ver también DIAMOND, 1975) nos da un modelo teórico en Aves que, en principio, parece también aplicable a los Saurios. Según este autor cuanto más similares sean las dietas en dos especies simpátridas, más diferentes serán los respectivos tamaños depredados y viceversa. Tenemos un ejemplo concreto en el caso de PS. algirus y $L$. lepida. De todos modos no podemos afirmar como HESPENHEIDE (op. cit.) que las diferencias en las categorías taxonómicas sean simple reflejo de preferencias de tamaño; en varias de nuestras especies hay un consumo «preferente» de Coleópteros con tamaños «preferidos» muy distintos para cada una de ellas (ver Tablas 5 y 6).

La interdependencia entre diversas dimensiones del nicho puede ponerse de manifiesto estudiando la correlación de sus valores de solapamiento. Entre la dimensión trófica y la temporal no existe más que una débil correlación positiva sin significación estadística $(r=0.28)$ que, sin embargo, indica que, en cierta medida, una dimensión depende de la otra. Esto es lógico pues, al margen de especializaciones concretas antes mencionadas, la depredación en intervalos horarios similares debe realizarse, en el caso de depredadores eclécticos, sobre grupos taxonómicos similares también, siempre que éstos no posean separaciones estrictas en la dimensión espacial. El factor de la estrategia de caza utilizada no se revela de mayor importancia máxime existiendo una dificultad creciente para separar de forma categórica las dos estrategias de caza habitualmente enunciadas como de «forrageo activo» y «sentarse y esperar».

Entre la dimensión espacial y la trófica la correlación resulta negativa $(r=-0.12)$ así como entre la espacial y la temporal $(r=-0.17)$ 

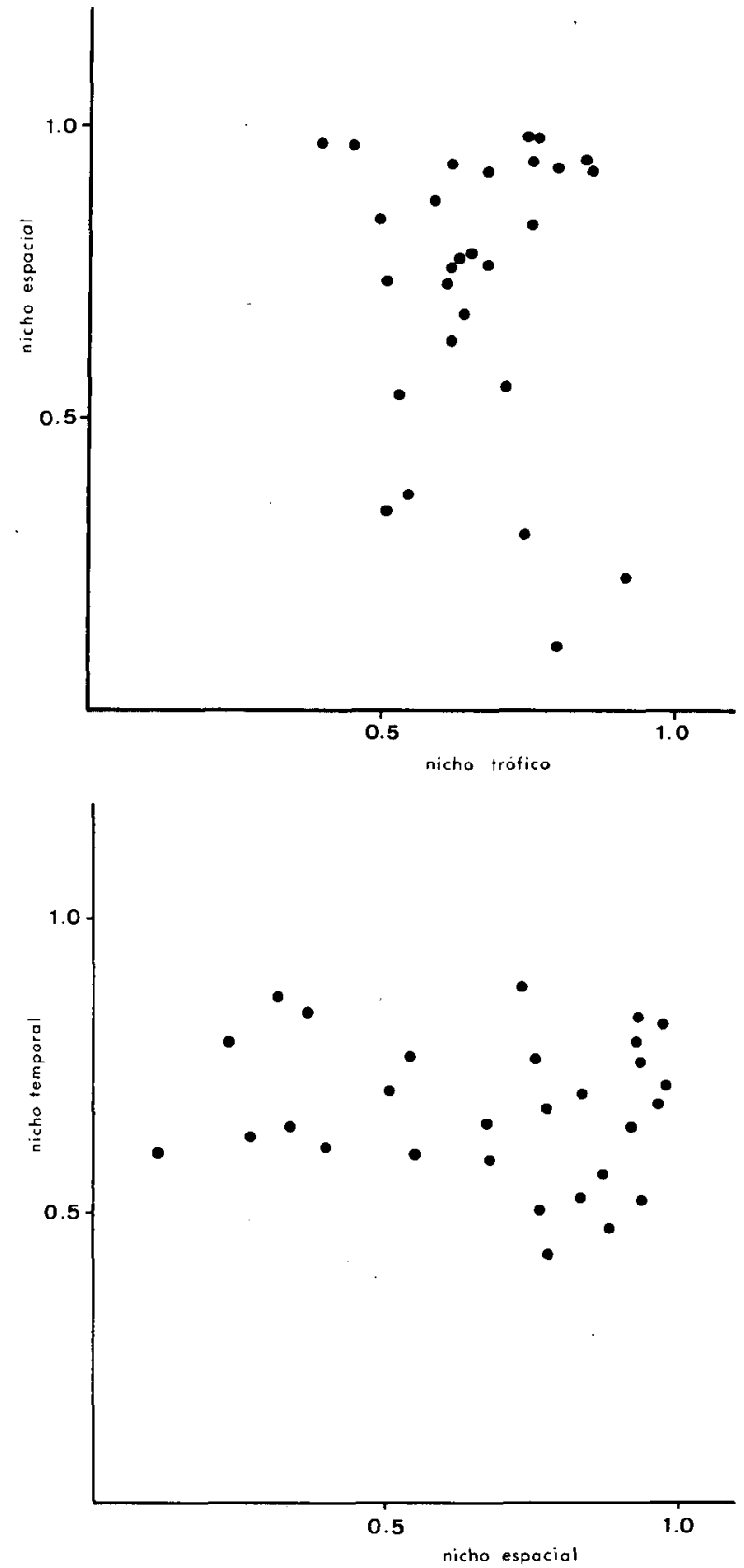

Figura 4. - Correlación entre los solapamientos de las dimensiones del nicho 
sin significación estadística en ambos casos. Sin embargo implica que en ciertas situaciones las especies de ritmo de actividad relativamente similar ocupan nichos espaciales algo diferentes o bien, si ocupan similares sustratos, poseerán regímenes alimenticios distintos (ver Figura 4).

MELLADO et. al. (1975), SCHOENER (1974) y otros autores llegan a resultados similares si bien incidiendo más concretamente en la importancia de la dimensión espacial. La matización sería que dicha dimensión produce, en efecto las más acusadas segregaciones, pero en íntima conexión con las otras dos dimensiones que, en el caso de los ecosistemas terrestres de la región mediterránea parecen adquirir aún mayor importancia. Desde nuestro punto de vista no existe pues en la estructura analizada ninguna dimensión que pueda considerarse como secundaria.

\section{BIBLIOGRAFÍA}

ARNOLD, E. N. \& BURTON, J. A. (1978) A Fiel Guide to the Reptiles and Amphibians of Britain and Europe. London Collins.

BALLINGER, R. E. \& BALLINGER, R. A. (1979) Food resource utilization during periods of low and high food availability in Sceloporus jarrovi (Sauria, Iguanidae). The Southwestern Nat. 24 (2): 347-363.

BARBAULT, R. (1977) Etude comparative des cycles journaliers d'activité des Lézards Cophosaurus texanus, Cnemidophorus scalaris et Cnemidophorus tigris dans le désert de Mapimi (Méxique). Bull. Soc. Zool. France 102: 159-168.

BARBAULT, R.; GRENOT, C. \& URIBE, Z. (1978) Le partage des ressources alimentaires entre les espèces de Lézards du désert de Mapimi (Méxique). La Terre et la Vie 32: 135-150.

DIAMOND, J. M. (1975) Assembly of species Communities, in: Ecology and Evolution of Communities (eds. M. L. Cody y J. M. Diamond). The Belknap Press of Harvard Univ. Press. London.

HEATWOLE, H. (1977) Habitat selection in Reptiles, in: Biology of the Reptilia (eds. C. Gans y D. W. Tinkle). Acad. Press. New York. 
HESPENHEIDE, H. A. (1971) Food preference and the extent of overlap in some insectivorous birds, with special reference to the Tyrannidae. Ibis 113: 59-72.

HESPENHEIDE, H. A. (1975) Prey Characteristics and Predator Niche Widht, in: Ecology and Evolution of Communities (eds. M. L. Cody y J. M. Diamond). The Belknap Press of Harvard Univ. Press. 158-180.

HUTCHINSON, G. E. (1975) Concluding Remarks. Cold spring Harbor Symp. Quant. Biol. 22: 415.

LEGLER, J. M. (1977) Stomach flusching: a technique for chelonian dietary studies. Herpetologica 33 (3): 281-284.

LEGLER, J. M. \& SULLIVAN, L. J. (1979) The Application of stomach flushing to lizards and anurans. Herpetologia 35 (2): 107-110.

LEVINS, R.(1968) Evolutions in changing environments. Princ. Univ. Press. Princenton, N. J.

LUIS CALABUIG, E. (1976) Ecosistemas de pastizal. Estudio de la vegetación mediante técnicas de análisis factorial. Crecimiento primario. Tesis doctoral. Univ. de Salamanca.

MACARTHUR, R. \& PIANKA, E. R. (1966) On optimal use of a patchy environment Amer. Nat. 100: 603-609.

MACARTHUR, R. \& LEVINS, R. (1967) The limiting similarity, convergence, and divergence of coexisting species. Amer. Nat. 101: 377-385.

MARGALEF, R. (1958) Informations theory in ecology. Gen. Syst. 3: 36-71.

MAY, R. M. (1974) On the theory of niche overlap. Theor. Pop. Biol. 5: 297-332.

MELLADO, J.; AMORES, F.; PARREÑO, F. F. e HIRALDO, F. (1975) The structure of a mediterranean lizard community. Doñana Acta Vert. 2 (2): 145-160. 
MELLADO, J. (1980) Utilización del espacio en una comunidad de lacértidos del matorral mediterráneo en la Reserva Biológica de Doñana. Doñana Acta Vert. 7: (1): 41-59.

OLIVER-MOSCARDÓ, S. y LUIS-CALABUIG, E. (1979) Factores termopluviométricos, in: Estudio multidisciplinario e integrado de la dehesa salmantina 1 . Estudio fisiográfico y descriptivo ( $3 .^{\circ}$ fasc.) 101-155.

ORIANS, G. H. y HORN, H. S. (1969) Overlap in food and foraging of four species of blackbirds in the potholes of central Washintong. Ecology 50: 930-938.

PÉREZ-MELLADO, V. (1981) La Lagatija de Bocage, Podarcis bocagei (Seoane, 1884): Primeros datos sobre su distribución, colorido y ecología. Amphibia Reptilia 3/4: 253-268.

PÉREZ-MELLADO, V. (1982) Los Lacertidae del Oeste del Sistema Central. Resúmenes de Tesis doctorales. Servicio de Pu. de la Univ. de Salamanca.

PÉREZ-MELLADO, V. (en prensa) Datos sobre Lacerta monticola Boulenger, 1905 (Sauria, lacertidae) en el Oeste del Sistema Central. Doñana Acta Vert.

PÉREZ-MELLADO, V. (en prensa) Alimentación de dos especies simpátridas de Saurios en el Sistema Central, Podarcis hispanica (Steindachner, 1870) y Podarcis bocagei (Seoane, 1884) (Sauria, Lacertidae). Studia Oecologica.

PIANKA, E. R. (1969) Sympatry of desert lizards (Ctenotus) in western Australia. Ecology 50: 1.020-1.030.

PIANKA, E. R. (1974) Niche overlap and diffuse competition. Proc. Nat. Acad. Sci. USA 71: 2.141-2.145.

PIANKA, E. R. (1978) Evolutionary Ecology. Harper Row. London.

PIELOU, E. C. (1966) The measurement of diversity in different types of biological collections. J. Theor. Biol. 13: 131-144. 
PIKE, G. H.; PULLIAM, R. H. \& CHARNOV, E. L. (1977) Optimal foraging: A selective review of theory and tests. Quart. Rev. Biol. 52: $137-154$.

RIVAS GODAY, S. (1955) Los Grados de vegetación de la Península Ibérica (con sus especies indicadoras). An. Inst. Bot. A. J. Cavanilles 13: 269-331.

RUIZ, X. \& JOVER, L. (1981) Tipificación trófica de poblaciones mediante estimas de dominancia y de la diversidad. Original mecanografiado de la comunicación presentada al XV Congreso Intern. de Fauna Cinegética y Silvestre. Trujillo (Cáceres).

SALVADOR, A. (1974) Guía de los anfibios y reptiles españoles. ICONA. Madrid.

SCHOENER, T. W. (1965) The evolution of bill size differences among sympatric congeneric species of birds. Evolution 19: 189-213.

SCHOENER, T. W. (1969) Models of optimal size for a solitary predator. Amer. Nat. 103: 277-313.

SCHOENER, T. W. (1971) Theory of feeding strategies. Ann. Rev. Ecol. Syst. 2: 369-404.

SCHOENER, T. W. (1974) Resource partitioning in ecological communities. Science 185: 27-39.

SCHMID, K. (1941) Vegetationsgürtel und Biozenose. Berichte der Schwetz Bot. Ges. Zurich.

SEVA, E. \& ESCARRÉ, A. (1980) Distribución espacial y temporal de Acanthodactylus erythrurus (Sauria, Lacertidae) en un arenal costero alicantino. Mediterránea 4: 133-162.

SHANNON C. E. (1948) A mathematical theory of communication. Bell. Systems Tech. J. 27: 379-423 y 623-656.

VALVERDE, J. A. (1967) Estructura de una comunidad de vertebrados terrestres. Monografías del C.S.I.C. n. ${ }^{\circ}$ 76: 1-218. Madrid. 